\title{
BMJ Open Can inspiratory muscle training improve weaning outcomes in difficult to wean patients? A protocol for a randomised controlled trial (IMweanT study)
}

Mariana Hoffman, ${ }^{1,2,3}$ Marine Van Hollebeke, ${ }^{2,3}$ Beatrix Clerckx, ${ }^{2,3}$ Johannes Muller, $^{3}$ Zafeiris Louvaris, ${ }^{2,3}$ Rik Gosselink, ${ }^{2,3}$ Greet Hermans, ${ }^{4,5}$ Daniel Langer ${ }^{2,3}$

To cite: Hoffman M, Van Hollebeke M, Clerckx B, et al. Can inspiratory muscle training improve weaning outcomes in difficult to wean patients? A protocol for a randomised controlled trial (IMweanT study). BMJ Open 2018;8:e021091. doi:10.1136/ bmjopen-2017-021091

- Prepublication history and additional material for this paper are available online. To view these files, please visit the journal online (http://dx.doi. org/10.1136/bmjopen-2017021091).

$\mathrm{MH}$ and MVH contributed equally.

Received 11 December 2017 Revised 26 March 2018 Accepted 18 April 2018

Check for updates

For numbered affiliations see end of article.

Correspondence to

Dr Mariana Hoffman; mariana_hoffmanb@yahoo. com.br

\section{ABSTRACT}

Introduction Respiratory muscle dysfunction has been associated with failure to wean from mechanical ventilation. It has therefore been hypothesised that these patients might benefit from inspiratory muscle training (IMT). Evidence, however, is thus far limited to data from small, single-centre studies with heterogeneity in inclusion criteria, training modalities and outcomes. The aim of this study is to evaluate the effects of a novel IMT method on weaning outcomes in selected patients with weaning difficulties.

Methods This study is designed as a double-blind, parallel-group, randomised controlled superiority trial with 1:1 allocation ratio. Patients with weaning difficulties will be randomly allocated into either an IMT group (intervention) or a sham-IMT group (control). Ninetypatients (45 in each group) will be needed to detect a $28 \%$ difference in the proportion of weaning success between groups (estimated difference in primary outcome based on previous studies) with a risk for type I error $(\alpha)$ of $5 \%$ and statistical power $(1-\beta)$ of $80 \%$. Patients will perform four sets of 6-10 breaths daily against an external load using a tapered flow resistive loading device (POWERbreathe KH2, HaB International, UK). Training intensity in the intervention group will be adjusted to the highest tolerable load. The control group will train against a low resistance that will not be modified during the training period. Training will becontinued until patients are successfully weaned or for a maximum duration of 28 days. Pulmonary and respiratory muscle function, weaning duration, duration of mechanical ventilation, ventilator-free days and length of stay in the intensive care unit will be evaluated as secondary outcomes. X2 tests and analysis of covariance with adjustments for baseline values of respective outcomesas covariates will be used to compare results after the intervention period between groups.

Ethics and dissemination Ethics approval was obtained from the local ethical committee (Ethische Commissie Onderzoek UZ/KU Leuven protocol ID: S60516). Results from this randomised controlled trial will be presented at scientific meetings as abstracts for poster or oral presentations and published in peerreviewed journals.
Strengths and limitations of this study

- This study will investigate the effects of inspiratory muscle training in a specifically selected group of difficult-to-wean patients and is designed as a randomised controlled trial with a sham treatment group with both patients and outcome assessors blinded to group allocation.

- Patients with weaning difficulties will be uniformly identified in accordance with a recently introduced and highly standardised classification system for weaning difficulties.

- Recruitment and monitoring will be facilitated by the use of an electronic record of ventilator management and weaning progression.

- A novel method for respiratory muscle training (tapered-flow resistive loading) will be applied.

- A limitation of the study is its single-centred design, which will limit external validity of the collected data.

Trial status Enrolment into the study have started in August 2017. Data collection and data analysis are expected to be completed in September 2021. Trial registration number NCT03240263.

\section{INTRODUCTION}

\section{Background and rationale}

Failure to wean is a complex problem with significant clinical and economic impact. ${ }^{1}$ The aetiology of weaning failure is usually multifactorial. Underlying severe respiratory disease with related pulmonary mechanical derangements, respiratory muscle dysfunction, heart failure, metabolic and endocrine disorders and cognitive dysfunction can all contribute to weaning failure. ${ }^{1-3}$ Weaning failure can partly be attributed to an imbalance between the ventilatory demand imposed on respiratory muscles and the capacity of the respiratory system 
to generate sufficient driving pressure to maintain tidal breathing. ${ }^{45}$ Respiratory muscle dysfunction in mechanically ventilated patients is highly prevalent and can result from inactivity that leads to muscle atrophy and decreased protein synthesis, neuromyopathy, hyperinflation, metabolic disorders, reduced oxygen delivery and possibly hypercapnia. ${ }^{6}$ In addition to ventilator-induced inactivity of the respiratory muscles, general impairments in neuromuscular function also frequently develop in patients admitted to the intensive care unit (ICU). This so-called ICU-acquired weakness contributes further to both peripheral and respiratory muscle dysfunction. ${ }^{78}$ Weaning from mechanical ventilation should be considered as early as possible to avoid further muscle atrophy, deconditioning and complications caused by the absence of spontaneous breathing. ${ }^{9}$ However, this is a challenging process and requires considerable effort. ${ }^{10}$

Intermittent spontaneous breathing periods (eg, using partially assisted modes of ventilation or spontaneous modes) are frequently applied during mechanical ventilation and may protect the respiratory muscles from the deleterious effects of prolonged inactivity. ${ }^{11} 12$ Limited data are also available showing that specific resistance training of the inspiratory muscles (IMT) can improve both respiratory muscle function and weaning outcomes. ${ }^{13-15}$ Available data indicate that the intervention is feasible and safe to apply in mechanically ventilated patients. From a recent meta-analysis summarising results from several small, single-centre studies, it appeared that benefits were more evident in patients that had already failed an attempt to wean. ${ }^{16}$

The studies performed so far on IMT in mechanically ventilated patients were however heterogeneous with regard to specific inclusion criteria, training modalities and outcomes evaluated. Not all studies specifically focused on patients with known weaning difficulties and notall studies evaluated weaning related outcomes. Timing of inclusion of patients was also not consistent between studies. Finally, most of those randomised controlled trials (RCTs) used a mechanical threshold loading (MTL) device for IMT which might not offer the ideal loading characteristics in this specific setting. ${ }^{16}$

In the current project, several key elements will be implemented that are hypothesised to improve the quality of the collected data. First, the focus will specifically be on patients with known weaning difficulties. These are the patients that are likely to benefit the most from an IMT intervention during mechanical ventilation. ${ }^{16}$ Second, IMT will be initiated as soon as weaning difficulties have been acknowledged. The classification of weaning difficulties will be performed in accordance with the most recent clinical definitions. ${ }^{17}$ Third, our main outcomes will be weaning success and other weaning-related parameters such as weaning duration. Finally, an alternative, potentially more optimal way of loading the respiratory muscles during the IMT sessions will be applied. This tapered flow resistive loading (TFRL) IMT, which is similar to isokinetic loading applied to limb muscles, is better adapted to the length-tension characteristics of the inspiratory muscles than the isotonic muscle loading applied during MTL. This characteristic will allow larger tidal volumes to be achieved during IMT for a giving external resistance. ${ }^{18}$ We hypothesise that, in analogy with data presented previously in patients with chronic obstructive pulmonary disease (COPD), this will result in better tolerance of higher training intensities with subsequent larger improvements in respiratory muscle function in comparison to MTL. ${ }^{18}$

Our primary hypothesis is that high-intensity IMT will improve weaning outcomes in difficult-to-wean patients.

\section{Objective}

This study will evaluate the effects of high-intensity inspiratory muscle strength training with TFRL in comparison with a sham, low-intensity inspiratory muscle endurance training on weaning outcomes in difficult-to-wean patients in the ICU included immediately after weaning difficulties have been identified.

\section{Trial design}

This trial is designed as a parallel-group, randomised controlled superiority trial with $1: 1$ allocation ratio. Participants and outcome assessors will be blinded to group allocation. The study was registered in a publicly accessible clinical trial database (clinicaltrials.gov identifier: NCT03240263). All procedures in this study will be performed in accordance with the ethical standards of the institutional review board of the UZ/KU Leuven and with the 1964 Helsinki declaration and its later amendments. Ethics approval was obtained from the responsible local ethical committee (Ethische Commissie Onderzoek UZ/KU Leuven protocol ID: S60516). The protocol is reported according to the Standard Protocol Items: Recommendations for Interventional Trials (SPIRIT) (see checklist online supplemental material). ${ }^{19}$

\section{METHODS}

\section{Patient and public involvement}

Patients were not involved in the study design or conduct of the study. At this stage, we have not yet decided as to how to disseminate the results to study participants.

\section{Study setting}

The trial will be conducted on both the surgical and medical ICU in a single centre (University Hospital Leuven, Belgium).

\section{Eligibility criteria}

Patients will be eligible if they have undergone a separation attempt and they have not been successfully weaned within 24 hours of this first separation attempt. The decision to start weaning and perform a separation attempt will be made by the clinical team caring for the patient. They will evaluate patients' 'readiness to wean' on a daily basis. In general, the following guidelines will be used when determining weaning readiness: (1) resolution 
of the acute phase of the disease for which the patient was intubated, (2) adequate oxygenation $\left(\mathrm{PaO}_{2}\right.$ arterial oxygen- $-\mathrm{PaO}_{2} / \mathrm{FiO}_{2}$ of $150-200$ requiring positive end-expiratory pressure (PEEP) $\leq 5$ to $8 \mathrm{cmH}_{2} \mathrm{O}$ and fraction of inspired oxygen $-\mathrm{FiO}_{2} \leq 0.4$ to 0.5 ), (3) absence of fever (temperature $<38^{\circ} \mathrm{C}$ ), (4) haemodynamic stability (eg, heart frequency $\leq 140 \mathrm{bpm}$ ), (5) stable blood pressure (BP), no or minimal vasopressors (dobutamine $\leq 5 \mu \mathrm{g}$ / $\mathrm{kg} / \mathrm{min}$, norepinephrine $\leq 0.1 \mu \mathrm{g} / \mathrm{kg} / \mathrm{min}$ ), (6) absence of myocardial ischaemia, (7) adequate haemoglobin (eg, haemoglobin $>7-10 \mathrm{~g} / \mathrm{dL}$ ), (8) adequate mentation and (9) adequate cough. ${ }^{90}$ These criteria can be individualised at the treating physicians' discretion. If the overall assessment is positive, a separation attempt will be performed. Separation attempts and weaning success will be defined in accordance with a recently developed classification system for weaning outcomes Weaning according to a New Definition (WIND). ${ }^{17}$

A separation attempt is defined differently for intubated patients and tracheotomised patients. For tracheotomised patients, a separation attempt is defined as 24 hours or more with spontaneous ventilation through a tracheostomy without any mechanical ventilation. For intubated patients, a separation attempt is a spontaneous breathing trial (SBT) with or without extubation, or an extubation directly performed without identified SBT (planned or unplanned extubation). An SBT can be performed either with the use of a T-tube, low-level pressure support ventilation $\left(\leq 8 \mathrm{cmH}_{2} \mathrm{O}\right)$ or continuous positive airway pressure $\left(\leq 5 \mathrm{cmH}_{2} \mathrm{O}\right)$ and has a duration of 30-120 min. ${ }^{171721}$ The SBT will be immediately interrupted in case of poor tolerance.

Criteria to evaluate the outcome of the SBT are (1) adequate gas exchange $\left(\mathrm{SpO}_{2} \geq 85 \%-90 \%, \mathrm{PaO}_{2}\right.$ $\geq 55-60 \mathrm{~mm} \mathrm{Hg}, \mathrm{pH} \geq 7.32$ and increase $\mathrm{PaO}_{2} \leq 10 \mathrm{~mm} \mathrm{Hg}$ ), (2) adequate ventilatory pattern (respiratory rate $\leq 30-35$ / min, $\Delta$ respiratory rate $<50 \%$ and Shallow Breathing Index between 60 breaths/min/L and 106 breaths/ $\mathrm{min} / \mathrm{L}$ ), (3) haemodynamically stable (heart rate $<120$ $140 /$ min, $\Delta$ heart rate $<20 \%$, systolic blood pressure (BP) $<180-200 \mathrm{~mm} \mathrm{Hg}$ and $>90 \mathrm{~mm} \mathrm{Hg}$ and $\Delta \mathrm{BP}<20 \%)$ and (4) subjective clinical signs (no changes in mental wellbeing and comfortable, no sweating, no use of accessory respiratory muscles, no paradoxical breathing). These criteria can be individualised by the treating physician who will decide whether or not to extubate the patient. If deemed appropriate in specific situations however, the treating physician may decide to prefer gradual reduction of Pressure Support (PS) or extubation without a prior SBT. In case a patient had a successful SBT without extubation, the reason why the patient was not extubated will be recorded and the training will be continued for maximum of 28 days or until successful weaning has been achieved. In general, SBTs are performed daily unless contraindicated. Reasons for not performing an SBT will be recorded in the patient's data record.

Difficult-to-wean patients, in whom no termination of weaning is achieved within 24 hours after a first unsuccessful separation attempt (and in whom no other rapidly reversible cause such as fluid overload is identified by the treating physician) and who are able to follow simple verbal commands necessary for the training, will be considered eligible for participation in the study.

The following exclusion criteria have been defined: pre-existing neuromuscular disease, haemodynamic instability (arrhythmia, decompensated heart failure, coronary insufficiency), haemoptysis, spinal cord injury above T8, use of any type of home mechanical ventilatory support prior to hospitalisation, any skeletal pathology that impairs chest wall movements such as severe kyphoscoliosis, congenital deformities or contractures, poor general prognosis or anticipated fatal outcome. ${ }^{20}$

\section{Recruitment}

Recruitment will be facilitated by the use of an electronic record (MetaVision, iMD-Soft, Needham, Massachusetts, USA) of the patient's SBTs and ventilation status completed by nurses and the treating physician on a daily basis. This electronic record will permit identification of difficult to wean patients at the earliest possible moment for inclusion according to the prespecified criteria. Researchers and physiotherapists will have access to this electronic record daily to ensure that eligible patients can be identified and informed about the possibility to participate in the study (figure 1).

\section{Sequence generation, randomisation, allocation concealment mechanism and blinding}

After providing written informed consent, patients will be randomly assigned to one of the two study groups: strength+endurance training group (intervention) or sham-endurance training group (control). Forty five patients will be included in each group (see figure 2) ${ }^{22}$

Opaque sealed envelopes will be prepared and sequentially numbered by a researcher not involved in the study ( 80 for the intervention group and 80 for the control group). Study groups will be also stratified for two factors that are known to influence weaning success rates: score on the Acute Physiology and Chronic Health Evaluation II scale (APACHE II) on admission and presence of COPD. The four piles of 40 sequentially numbered envelopes (containing 20 intervention and 20 control each) should contain a sufficient number of envelopes in each stratum. Patients will be grouped and randomised according to the following characteristics: Group 1, APACHE score < 18 and COPD; Group 2, APACHE score $>18$ and COPD; Group 3, APACHE score $<18$ and non-COPD; and Group 4, APACHE score $>18$ and non-COPD. ${ }^{22}$ Block randomisation with random block sizes of 4 and 6 will be performed. After identifying and characterising candidates for the study, patients will be allocated into either intervention or control group by opening randomisation envelops from one of the four corresponding strata sequentially (from lowest to next highest number).

Physiotherapists performing the training cannot be blinded for the treatment; however, patients, nurses, 




Figure 1 Template of the electronic record (MetaVision, iMD-Soft) where information regarding eligibility criteria can be recorded. Data regarding ventilatory mode, spontaneous breathing trial (SBT) performance, SBT failure or success, tidal volume, fraction of inspired oxygen $\left(\mathrm{FiO}_{2}\right)$, positive end-expiratory pressure (PEEP), tidal volume and pressure support value are recorded in the system every $30 \mathrm{~min}$. Continuous positive airway pressure (CPAP), pressure support (PS), assisted spontaneous breathing (ASB), automatic tube compensation (ATC).

treating physicians and outcome assessor will be blinded to the group allocation. Training will be scheduled in consultation with the treating team and nurses and physicians will be asked not to enter the room during the training unless in case of emergency. Participants will be unblinded and informed about their group allocation after completion of the trial. Patients who were allocated to the control group will be offered strength training with the TFRL device on completion of the trial. A blinded analyst will perform the statistical analyses.

\section{Interventions}

Inspiratory muscle training

Training will be performed under full supervision of physiotherapists on the ICU. The physiotherapists will monitor dose of training, amount of work performed and patient's symptoms during the sessions. Patients will be placed in a semiupright sitting position in bed (head board at 45 degrees), aspirated if necessary, and the tracheostomy or tube cuff will be sufficiently inflated in order to prevent potential air leakage during IMT sessions. Before, during and after every session, vital parameters (respiratory rate, heart rate, oxygen haemoglobin saturation and BP) will be recorded. Patients will be instructed to perform fast and forceful inspirations against the inspiratory resistance and to achieve a full inspiration and expiration at every breath. Instructions and encouragements during the sessions will be standardised. Visual feedback will be available on a laptop screen to provide patients information on their performance provided by the training software (BreatheLink Software, HaB International, UK)during the training (volume and flow generated during each breath and total number of inspiratory efforts). The training sessions will be interrupted if patients report intolerable symptoms of dyspnoea or breathing discomfort, when the transcutaneous oxygen desaturation falls below $85 \%$ or when patients starts to cough. Sessions will consist of four sets of 6 to 10 breaths per set with resting periods of at least $2 \mathrm{~min}$ between sets. Patients in the intervention group will perform the training using the TFRL device (POWERbreathe KH2, HaB International, UK). In order to maximise work of breathing performed during the training sessions, the resistance in the intervention group will be adjusted on a daily basis to the highest tolerable load. The external load will be chosen to correspond with the highest possible percentage of maximal inspiratory mouth pressure (PImax) (typically between $30 \%$ and $50 \%$ PImax that will still allow inspiratory tidal volume expansion to at least $70 \%$ of the patient's inspiratory vital capacity. In addition to the ability to perform full vital capacity inspirations, the choice of the highest tolerable load will also depend on the symptom scores of patients. The respiratory effort scores reported by patients after the training sessions should ideally range between 4 and 6 on a modified Borg scale. Patients in the control group will perform the training against an external load that will be set to represent a maximum of $10 \%$ of their 




Figure 2 Flowchart of the study design. Blood pressure (BP); Heart rate (HR); Intensive care unit (ICU); Maximal inspiratory pressure (PImax) ; Peak inspiratory flow (PIF), ; Respiratory rate (RR); Rapid Shallow Breathing index (RSBI); Haemoglobin saturation $\left(\mathrm{SpO}_{2}\right)$; Vital capacity (VC). 
PImax) with no adjustments to this load during the entire training period. Training in the control group will also be performed with the TFRL device if the training load ( $10 \%$ PImax) is equal to or higher than $3 \mathrm{cmH}_{2} \mathrm{O}$. Otherwise, if the training load needs to be adjusted to less than $3 \mathrm{cmH}_{2} \mathrm{O}$ (ie, in case this minimal resistance corresponds to more than $10 \%$ PImax), an adapted device that is able to provide a lower load (POWERbreathe Classic, Hab International, UK) will be used. ${ }^{23}$

After each session, scores on perceived breathing effort and dyspnoea will be recorded with a modified Borg CR-10 scale. ${ }^{18} 20$ Training will be continued for a maximum duration of 28 days or until the patient is successfully weaned from mechanical ventilation. ${ }^{20}$ Patients still on mechanical ventilation after 28 days of IMT or who die during this period will be considered 'failure to wean'. In patients who will be transferred to other hospitals during this period registration of the weaning process will be continued. We will also make an attempt to continue the assigned intervention if feasible.

Weekly measurements of PImax, forced vital capacity (FVC) and peak inspiratory flow (PIF) will also be used to optimise training resistance. ${ }^{20}$

\section{Common therapeutic interventions applied in both study arms}

All patients will receive strict glycaemic control, enteral feeding as soon as possible, no supplemental parenteral nutrition in the first week of ICU stay ${ }^{24}$ and a standardised early mobilisation protocol. ${ }^{25}{ }^{26}$ Sedation is titrated on a daily basis to the minimum dose needed for patient comfort unless presence of medical conditions requires heavy sedation (eg, severe acute respiratory distress syndrome (ARDS), intracranial hypertension, etc.). All patients will follow the institutional protocol for early mobilisation entitled 'Start to Move ASAP' ${ }^{25}$ This protocol involves passive and active mobilisation according to the individual patient's clinical status including different postures or activities such as sitting in bed, sitting in the chair, cycling or walking. ${ }^{25}$

\section{Outcomes}

Primary outcome

The primary outcome of this study is weaning success. Successful weaning is defined in intubated patients as extubation without death or reintubation within the next 7 days whether postextubation non-invasive ventilation is used or not, or ICU discharge without invasive mechanical ventilation within 7 days. For tracheotomised patients, weaning is considered successful when a patient is on spontaneous ventilation through tracheostomy without any mechanical ventilation during 7 consecutive days or discharged with spontaneous breathing, whichever comes first (table 1). ${ }^{17}$

\section{Secondary outcomes}

Secondary outcomes are: duration of the weaning process (days since first separation attempt until successful weaning), length of stay in the ICU (days), duration of mechanical ventilation (days since patient was connected to mechanical ventilator), Rapid Shallow Breathing Index (breaths/min/L), VC (L), PImax $\left(\mathrm{cmH}_{2} \mathrm{O}\right)$ and ventilator-free days (days free from the mechanical ventilation within the treatment period (from start of IMT until maximum of 28 days after enrolment) (table 1 ).

\section{Assessment methods}

PImax will be measured after endotracheal aspiration, with the patient positioned in a semiupright sitting position in bed, using a unidirectional valve that will be attached to the patient's tracheostomy tube or endotracheal tube. The valve allows exhalation but occludes inspiration. Patients will breathe through this valve and will be encouraged to inhale as forcefully as possible during each inspiratory attempt for an uninterrupted period of $25 \mathrm{~s}^{2027}$ The valve is connected to a manometer (PFT Systems Pocket-Spiro) and to a computer that displays pressure/time plots generated during the manoeuvres. PImax measurements will be performed three times with $2 \mathrm{~min}$ rest in between manoeuvres. The highest value of the 1 second plateau pressure generated on one of the manoeuvres will be considered for analysis.

Assessments of FVC will be performed using a spirometer (PFT Systems Pocket-Spiro) connected to the tracheostomy tube or endotracheal tube. After full inspiration the patient will be asked to perform a maximal forced expiration all the way to Residual Volume (RV) followed by a maximal inspiration until achievement of total lung capacity. The manoeuvres will also be displayed in a graphic format on the computer screen and values will be recorded. Duringthe FVC manoeuvre, other variables will be assessed and recorded: forced expiratory volume in one second $\left(\mathrm{FEV}_{1}\right)$, peak expiratory flow, $\mathrm{FEV}_{1} / \mathrm{FVC}$ and inspiratory capacity. Measurements of FVC will be repeated at least three times with a 2 min rest between the manoeuvres and the best manoeuvre (considering the reproducibility criteria of differences between manoeuvres not higher than 10\%) will be considered for analysis. ${ }^{20}$

PIF will also be assessed during the FVC maneuver and recorded using the same spirometer. Measurements of PImax, FVC and PIF will be performed weekly (table 1).

The RSBI will be measured daily, right before the IMT session during one minute of spontaneous breathing. The average value of RSBI at time of inclusion and before a successful separation attempt (after 24 hours for tracheotomised patients and at the end of a successful SBT for intubated patients) will be considered for evaluation and is calculated from the ratio of respiratory frequency to tidal volume. (table 1)

Weaning duration, ventilator-free days, length of stay and duration of mechanical ventilation will be collected after weaning success by retrieving data from the patients' electronic database record from the hospital (MetaVision, iMD-Soft) (table 1). 
Table 1 Trial time schedule-overview of interventions and assessments performed during the study period.

\section{Study period}

\begin{tabular}{|c|c|c|c|c|c|c|c|c|c|}
\hline Time point & $\begin{array}{l}\text { Enrolment } \\
\text { Day } 0\end{array}$ & $\begin{array}{l}\text { Allocation } \\
\text { Day } 0\end{array}$ & \multicolumn{6}{|c|}{ Postallocation } & $\frac{\text { Closeout }}{\text { Day } x}$ \\
\hline Informed consent & $\bullet$ & & & & & & & & \\
\hline Allocation & & $\bullet$ & & & & & & & \\
\hline $\begin{array}{l}\text { Sham endurance } \\
\text { (Daily IMT: } 4 \times 6-10 \text { breaths }\end{array}$ & & $\bullet$ & $\bullet$ & $\bullet$ & $\bullet$ & - & $\bullet$ & & \\
\hline
\end{tabular}

\section{Assessments}

MIP
RSBI
Weaning success
Weaning duration
Length of stay in ICU
Length of stay in hospital

- indicates performance of a measure on the day indicated. Arrows represent assessments or interventions that will be performed daily throughout the study period.

ICU, intensive care unit; IMT, inspiratory muscle training; MIP, maximal inspiratory pressure; RSBI, Rapid Shallow Breathing Index; SSA, successful separation attempt; VC, vital capacity.

\section{Participant timeline}

The time schedule for enrolment, interventions and assessments is presented in table 1 .

\section{Sample size calculation}

Based on previously observed proportions of weaning success in a subgroup of patients with weaning difficulties of $78 \%$ in an IMT group and 50\% in a control group, ${ }^{16} 20$ assuming $\alpha$ and $\beta$ risks of $5 \%$ and $20 \%$, a sample of 45 patients in the IMT group and 45 in the control group will be necessary to detect a significant difference between groups in our primary outcome: weaning success. ${ }^{1620}$

\section{Data management and monitoring}

The principal investigator will have access to all data of patients and training sessions and will be responsible for recollection, adjustments and supervision of training sessions with the physiotherapists of the ICU. The principal investigator will also try to assess outcome measures of included and allocated patients that refuse to continue the training before the end of training duration.

Data regarding ventilator management will be collected from the individual electronic patient records (MetaVision, iMD-Soft) as illustrated in figure 1. Data will be recorded for each patient in an excel data collection sheet and an example is included as a online supplementary file. The following information regarding patient characteristics will be collected: age, weight, height, gender, diagnosis, classification as difficult or prolonged weaning, reasons for weaning failure, date of the start of the weaning process, date of the first successful separation attempt, date of disconnecting mechanical ventilation, date of successful weaning and date of ICU discharge. Data on training parameters will furthermore be collected before, during and after individual IMT sessions on a daily basis. These include data on training volume and training intensity and tidal volumes achieved during training sessions. Borg scores on respiratory effort and dyspnoea after training as well as cardiac frequency, BP, respiratory rate and oxygen haemoglobin saturation before and after the training sessions will also be recorded. Information on ventilator settings, and data concerning SBT including reasons for not performing an SBT, as well as duration and breathing pattern during spontaneous breathing periods will also be recorded on a daily basis. The "start to move level' of each patient will also be registered. This score will reflect the level of general reconditioning exercises that each patient will have received on separate days during the intervention period.

Details of all training sessions (pressure, volume, power and external work) will also be recorded and saved in the 
training software (BreatheLink Software, HaB International, UK).

A person responsible for data management and monitoring will be chosen to supervise data entry during the study period prior to conduction of statistical analyses. Electronic records of patients and training sessions will be stored as a database in password-protected files and will be updated, saved and backed up regularly. Paper documents will be anonymised (every patient will be assigned with a different code), collected and stored in individually coded patient files. Weekly backup of data will be performed. All researchers will have access to the results of the analyses. Data will be coded to ensure confidentiality and digital information will be password protected.

\section{Monitoring: adverse events and withdrawal}

No serious adverse events have been reported to be caused by IMT. ${ }^{16}$ To ensure safety, patients will be monitored before and during the training sessions. Any adverse event occurring during the training interventions (whether related or unrelated to the intervention) will be registered and reported to the ICU medical staff and multidisciplinary team. Decisions to withdraw patients from the study will be made by the researcher blinded to allocation in consultation with the multidisciplinary team or, of course, by the patient himself. Reasons for withdrawal will be recorded.

\section{Statistical analysis}

For data analysis, a true intention-to-treat analysis will be performed on the primary outcome to compare weaning success between groups. Modified intention-to-treat analysis will be performed on secondary outcomes that are not lost to follow-up. Analysis of covariance will be used for secondary variables for comparison between groups before and after IMT since it permits comparison of outcomes between groups correcting for eventual baseline differences as a covariate. ${ }^{28}$ Statistical significance will be set at $\mathrm{p}<0.05$. Kaplan-Meier curves with $\log$ rank tests will be used to compare the time to successful extubation and liberation from mechanical ventilation. Patients who will die or who will be transferred to another unit during the intervention period will be censored for this analysis; rates of deaths in both study groups will be compared as a secondary outcome. Cox proportional hazards regression will furthermore be used to adjust this analysis for confounding factors that can affect MV duration.

Additional and adjusted analysis will be performed by intention-to-treat analysis and per protocol analysis. ${ }^{29} 30$

Outcomes not assessed in postintervention will be considered as lost for follow-up. In these cases, data will not be imputed and values will be labelled as missing data.

\section{Ethics and dissemination}

The local Ethic Commission approved this study protocol (S60516) and the results from the RCT will be submitted for publication. Any protocol amendments will be submitted to same local Ethic Commission and communicated to the trial registry. Written informed consent will be obtained from all patients if awake and adequate or from a family member if unconscious. The results arising from this RCT will be presented at scientific meetings as abstracts for poster or oral presentations and published in peer-reviewed journals. There is no intention of using a professional writer, and authorship will be based on the collaboration of each member of the research group.

\section{Author affiliations}

${ }^{1}$ Federal University of Minas Gerais, Rehabilitation Sciences Program, Belo Horizonte, Brazil

${ }^{2} \mathrm{KU}$ Leuven - University of Leuven, Department of Rehabilitation Sciences, Leuven, Belgium

${ }^{3}$ Department of Intensive Care Medicine, University Hospitals Leuven, Leuven, Belgium

${ }^{4}$ Medical Intensive Care Unit, Department of General Internal Medicine, University Hospitals Leuven, Leuven, Belgium

${ }^{5}$ Laboratory of Intensive Care Medicine, Division of Cellular and Molecular Medicine, KU Leuven, Leuven, Belgium

Contributors $\mathrm{MH}, \mathrm{BC}, \mathrm{DL}$ and $\mathrm{RG}$ are responsible for the overall development of an ethically sound protocol. MH, BC, DL, RG, MVH, ZL, GH and JM are involved in the conception and production of the study and the development of the initial protocol. All authors contributed to the drafting, critical revision and final approval of the document.

Funding MH was sponsored by Fundaç̃o de Amparo a Pesquisa de Minas GeraisFAPEMIG/Brazil grant numbers [309494/2013-3 and 442973/2014-4]. ZL is a postdoctoral fellow of the FWO-Flanders (Fellowship number 12U5618N).

Competing interests None declared.

Patient consent Not required.

Ethics approval Ethische Commissie Onderzoek UZ/KU Leuven - protocol ID: S60516.

Provenance and peer review Not commissioned; externally peer reviewed.

Open access This is an open access article distributed in accordance with the Creative Commons Attribution Non Commercial (CC BY-NC 4.0) license, which permits others to distribute, remix, adapt, build upon this work non-commercially, and license their derivative works on different terms, provided the original work is properly cited and the use is non-commercial. See: http://creativecommons.org/ licenses/by-nc/4.0/

(c) Article author(s) (or their employer(s) unless otherwise stated in the text of the article) 2018. All rights reserved. No commercial use is permitted unless otherwise expressly granted.

\section{REFERENCES}

1. Martin DA, Smith BK, Gabrielli A, et al. Diaphragm weakness and weaning: a rehabilitation perspective. Respir Physiol Neurobiol 2013;189:377-83.

2. Heunks LM, van der Hoeven JG. Clinical review: The ABC of weaning failure-a structured approach. Crit Care 2010;14:245.

3. Berger D, Bloechlinger S, von Haehling S, et al. Dysfunction of respiratory muscles in critically ill patients on the intensive care unit. J Cachexia Sarcopenia Muscle 2016;7:403-12.

4. De Jonghe B, Bastuji-Garin S, Durand MC, et al. Respiratory weakness is associated with limb weakness and delayed weaning in critical illness. Crit Care Med 2007:35:2007-15.

5. Vassilakopoulos T, Zakynthinos S, Roussos C. Respiratory muscles and weaning failure. Eur Respir J 1996;9:2383-400.

6. Dres M, Goligher EC, Heunks LMA, et al. Critical illness-associated diaphragm weakness. Intensive Care Med 2017;43:1441-52.

7. Stevens RD, Dowdy DW, Michaels RK, et al. Neuromuscular dysfunction acquired in critical illness: a systematic review. Intensive Care Med 2007;33:1876-91. 
8. De Jonghe B, Bastuji-Garin S, Sharshar T, et al. Does ICU-acquired paresis lengthen weaning from mechanical ventilation? Intensive Care Med 2004;30:1117-21

9. Boles JM, Bion J, Connors A, et al. Weaning from mechanical ventilation. Eur Respir J 2007;29:1033-56.

10. Tobin MJ. Advances in mechanical ventilation. N Engl J Med 2001;344:1986-96.

11. Gosselink R, Langer D. Recovery from ICU-acquired weakness; do not forget the respiratory muscles!. Thorax 2016;71:779-80.

12. Gayan-Ramirez G, Testelmans D, Maes K, et al. Intermittent spontaneous breathing protects the rat diaphragm from mechanical ventilation effects. Crit Care Med 2005;33:2804-9.

13. Schellekens WJ, van Hees HW, Doorduin J, et al. Strategies to optimize respiratory muscle function in ICU patients. Crit Care 2016;20:103.

14. Mohamed R, Basiouny H EL, Salem M. Response of Mechanically Ventilated Respiratory Failure Patients to Respiratory Muscles Training. Med. J. Cairo Univ 2014;82:19-24.

15. Pascotini $S$, Denard $C$, Nunes $O$, et al. Respiratory muscle training in patients weaning from mechanical ventilation. ABCS Health Sci 2013;39:12-16.

16. Elkins $M$, Dentice R. Inspiratory muscle training facilitates weaning from mechanical ventilation among patients in the intensive care unit: a systematic review. J Physiother 2015;61:125-34.

17. Béduneau G, Pham T, Schortgen F, et al. Epidemiology of Weaning Outcome according to a New Definition. The WIND Study. Am J Respir Crit Care Med 2017;195:772-83.

18. Langer D, Charususin N, Jácome C, et al. Efficacy of a Novel Method for Inspiratory Muscle Training in People With Chronic Obstructive Pulmonary Disease. Phys Ther 2015;95:1264-73.

19. Chan AW, Tetzlaff JM, Gøtzsche PC, et al. SPIRIT 2013 explanation and elaboration: guidance for protocols of clinical trials. $B M J$ 2013;346:e7586.
20. Martin AD, Smith BK, Davenport PD, et al. Inspiratory muscle strength training improves weaning outcome in failure to wean patients: a randomized trial. Crit Care 2011;15:R84.

21. Ladeira MT, Vital FM, Andriolo RB, et al. Pressure support versus T-tube for weaning from mechanical ventilation in adults. Cochrane Database Syst Rev 2014:CD006056.

22. Doig GS, Simpson F. Randomization and allocation concealment: a practical guide for researchers. J Crit Care 2005;20:187-91.

23. Fregni F, Imamura M, Chien HF, et al. Challenges and recommendations for placebo controls in randomized trials in physical and rehabilitation medicine: a report of the international placebo symposium working group. Am J Phys Med Rehabil 2010;89:160-72.

24. Casaer MP, Mesotten D, Hermans G, et al. Early versus late parenteral nutrition in critically ill adults. $N$ Engl $J$ Med 2011;365:506-17.

25. Gosselink R, Clerckx B, Robbeets C, et al. Physiotherapy in the intensive care unit. Netherlands Journal of Critical Care 2011;15:66-75

26. Gosselink R, Bott J, Johnson M, et al. Physiotherapy for adult patients with critical illness: recommendations of the European Respiratory Society and European Society of Intensive Care Medicine Task Force on Physiotherapy for Critically III Patients. Intensive Care Med 2008;34:1188-99.

27. Marini JJ, Smith TC, Lamb V. Estimation of inspiratory muscle strength in mechanically ventilated patients: The measurement of maximal inspiratory pressure. J Crit Care 1986;1:32-8.

28. Vickers AJ, Altman DG. Statistics notes: Analysing controlled trials with baseline and follow up measurements. BMJ 2001;323:1123-4.

29. Sedgwick P. Intention to treat analysis versus per protocol analysis of trial data. BMJ 2015;350:h681.

30. Abraha I, Cherubini A, Cozzolino F, et al. Deviation from intention to treat analysis in randomised trials and treatment effect estimates: meta-epidemiological study. BMJ 2015;350:h2445. 